\title{
Biotic and Abiotic Stress Responses in Crop Plants
}

\author{
Thomas Dresselhaus ${ }^{1, *(1)}$ and Ralph Hückelhoven ${ }^{2, *}$ \\ 1 Cell Biology and Plant Biochemistry, Biochemie-Zentrum Regensburg, University of Regensburg, \\ 93053 Regensburg, Germany \\ 2 Phytopathology, TUM School of Life Sciences Weihenstephan, Technical University of Munich, \\ 85354 Freising, Germany \\ * Correspondence: thomas.dresselhaus@ur.de (T.D.); hueckelhoven@wzw.tum.de (R.H.); \\ Tel.: +49-941-9433016 (T.D.); +49-8161-713682 (R.H.)
}

Received: 6 November 2018; Accepted: 15 November 2018; Published: 19 November 2018

check for updates

\begin{abstract}
Agricultural productivity depends on increasingly extreme weather phenomena, and the use of germplasm that has to be continuously improved by plant breeders to become tolerant to various biotic and abiotic stresses. Molecular plant biologists try to understand the mechanisms associated with stress responses and provide knowledge that could be used in breeding programs. To provide a partial overview about our current understanding about molecular and physiological stress responses, and how this knowledge can be used in agriculture, we have edited a special issue on "Biotic and Abiotic Stress Responses in Crop Plants". Contributions are from different fields including heat stress responses, stress responses during drought and salinity, as well as during flooding, and resistance and susceptibility to pathogenetic stresses and about the role of plant functional metabolites in biotic stress responses. Future research demand in particular areas of crop stress physiology is discussed, as well as the importance of translational research and investigations directly in elite crop plants and in the genetic resources available for breeding.
\end{abstract}

Keywords: heat and drought stress; salinity; flooding; plant immunity; cereals; secondary metabolites; signaling; stress hormones

\section{Introduction}

While the demands for crop products continue to increase, agricultural productivity is threatened by various stress factors, often associated with global warming [1-3]. In Germany, for example, precipitation has increased by $11 \%$ during the past 100 years by up to $30 \%$ in the cold season, while summers are much dryer and the number of days with temperatures above $30^{\circ} \mathrm{C}$ has doubled [4]. These conditions often favor pathogens and negatively affect plant productivity and fertility. Plants have to adapt in a physiologically costly way to these changing environmental conditions, resulting in reduced availability of resources to produce biomass, seeds, and thus yield. Moreover, the combination or alteration of diverse abiotic and biotic stress factors may further cause trade-off between plant responses that are appropriate for adaptation to one stress, but can enhance susceptibility to other stresses. Climate-driven migration of pathogens and pests further confronts locally adapted crop genotypes with new biotic stress factors. This clash of globally increasing yield demands with increasing yield-threatening environmental conditions asks for massive investments into plant stress resistance research and development. To sustain and improve yields, it is necessary to understand how plants respond to various stresses and to use the knowledge generated in modern plant breeding programs. Most knowledge regarding the molecular mechanisms associated with stress responses has been obtained from investigations using the model plant Arabidopsis thaliana. However, it is often unclear to which extent the elucidated mechanisms also operate in crops. 
Financed by the German Research Foundation DFG and by the Bavarian State Ministry of the Environment and Consumer Protection, molecular plant researchers in Bavaria (in southeast Germany) have now established two research consortia with the aim to elucidate the molecular mechanisms by which model and crop plants adapt to various biotic and abiotic stresses. The knowledge generated will then be used to develop crop plants better adapted to the consequences of global warming. Within the "BayKlimaFit" network (Strategies for the Adaptation of Crop Plants to Climate Change), seven groups study plant responses during flooding and cold as well as heat and drought stress, while two labs investigate symbionts and pathogens (for an overview, see http:/ / www.bayklimafit.de) [5]. The Collaborative Research Centre (CRC) SFB924 aims to understand Molecular Mechanisms Regulating Yield and Yield Stability in Plants (http://sfb924.wzw.tum.de) [6] and to transfer knowledge from model plants to crop plants or from one crop plant to another crop plant. Within the center, 12 groups investigate molecular mechanisms regulating abiotic and biotic stress responses in Arabidopsis and crop plants such as maize, barley, tomato, and Medicago. As knowledge generated by molecular plant researchers often does not reach plant breeders and decision-makers, we discussed with our colleagues the idea of editing a Special Issue about Biotic and Abiotic Stress Responses in Plants in the journal Agronomy, with the goal of contributing to filling this gap and additionally highlighting this exciting research area.

This Special Issue, therefore, collected contributions from groups of both research consortia, complemented by papers from colleagues worldwide, reporting how crop plant species respond to various abiotic stresses, such as drought, heat, flooding, and salinity, as well as biotic stimuli during microbial infections. It contains reviews, opinions, perspectives, and original articles, and its focus is on our molecular understanding of biotic and abiotic stress responses in crops, highlighting, among other aspects, the role of stress hormones, secondary metabolites, signaling mechanisms, and their regulation, as well as state-of-the-art omics approaches. Strategies and ideas to achieve stress tolerance and to maintain yield stability of agricultural crops during stress periods are also discussed. These include perspectives on how knowledge from model plants can be utilized to facilitate crop-plant breeding and biotechnology.

\section{Biotic Stress Responses and the Role of Secondary Metabolites}

Plants function in complex environments and are increasingly often considered not as individual genotypes, but as holobionts that function in genetic, physiological, and evolutionary units with their associated microbiota [7]. The microbiota can be beneficial, detrimental, or neutral for plant fitness and their function can also change depending on environmental challenges. In their report, Aslam and Ali used the halotolerant plant species Suaeda fruticosa (L.) for the identification of a variety of plant-associated bacterial genera. They isolated bacteria and performed physiological characterization of auxin biosynthesis capacities, biofilm formation, and halotolerance, amongst others. Astonishingly, several bacterial strains showed an ability to support the growth of corn plants under salt stress conditions, and this was partially associated with higher antioxidant capacities. This finding shows the potential of plant-associated microbiota for application as biologicals in plant protection against abiotic stress and suggests that bacteria can prime plants for higher cell protection potential [8].

Interaction of plants with microbes or microbe-associated molecular patterns can also induce resistance to secondary infections by pathogens. This involves the production and systemic signaling of a complex of low-molecular-weight plant metabolites, which are well described for dicotyledonous plants, but poorly understood for monocotyledonous plants such as cereal crops. Lenk and coworkers used resistance-inducing compounds from the model Arabidopsis thaliana and tested their potential in barley. They discovered a challenging-pathogen-dependent effect of individual compounds in inducing either disease resistance or susceptibility in barley [9]. This highlights the conservation and diversification of plant responses to stress signaling compounds when comparing dicots and monocots.

Stress-related metabolic alterations occur in plants in a genotype-specific manner. Therefore, metabolic changes can be used in targeted breeding for specific agronomic traits such as stress resistance 
or food functions. In this context, Dawid and Hille provide a review on functional metabolomics and the analytical techniques used in this discipline [10]. They describe how metabolite profiles can be translated into stress resistance or desired sensory traits of the crop plant, but also discuss the need for orchestrated biological and structural characterizations of unidentified marker metabolites.

Benzoxazinoids (BXs) are secondary plant metabolites from grasses with a high potential for the chemical defense against biotic stress factors from diverse kingdoms of life. Niculaes and coauthors provide a comprehensive overview of the biosynthesis, metabolism, and biological activities of BXs [11]. They report on the broad spectrum of biological functions of BXs, for example, from toxic to insect-health-promoting effects. Together, BXs belong to the best-characterized chemical defense compounds in maize and are of strong agronomic relevance. However, their exact biosynthesis pathways and functions in other Poaceae crops and their wild relatives appear to be not well understood. Future research demand is accordingly discussed.

Disease resistance and susceptibility are two sides of the same coin, aren't they? Two perspective articles in this Special Issue discuss how to make use of the recently expanding knowledge on how plants recognize and defend microbial intruders and how microbial pathogens trick the plant to overcome immunity and manipulate host functions for their own demand. Quantitative broad-spectrum immunity against microbial pathogens depends to a substantial extent on the efficient recognition of nonspecific microbe-associated molecular patterns and host-derived endogenous damage-associated molecular patterns. Ranf explains the mechanism of the sensitive detection of these molecules by cell-surface pattern recognition receptors (PRRs) [12]. She reports the great progress in the recent identification of novel PRRs from plants, with most of them being identified in Arabidopsis thaliana. However, she also shows the perspective for the translation of this knowledge into biotechnological approaches that will allow the establishing, enhancing, and even designing of pathogen pattern-sensing capacities in model and crop plants.

Engelhardt and coauthors discuss that disease susceptibility is more than just the failure of plant immunity, and involves so-called susceptibility (S) factors of the host plant [13]. They highlight physiological functions of $S$ factors, give examples of how pathogen effectors manipulate $S$ factors, and discuss whether and how we can use them by modern targeted breeding approaches, including guided endonuclease technology, to turn susceptibility into resistance. Furthermore, the authors discuss the natural occurrence, possible trade-offs, and durability of loss of susceptibility.

\section{Abiotic Stress Responses and the Usage of RNA-seq Methodology}

A number of papers consider the effect of heat and drought stress on crops. Nadeem and coworkers review the diverse effects of heat stress on crop yield [14]. They report that heat stress is especially deleterious to certain developmental stages, including the generation and function of reproductive organs. Physiological plant responses involve mechanisms to prevent membrane damage and to regulate photosynthesis, respiration, and transpiration. Molecular heat stress responses involve ROS, NO, and $\mathrm{Ca}^{2+}$ signaling as well as the induction of heat stress factor genes (HSFs) and other transcription factors, which is discussed to be used in future approaches to engineer tolerant crops. Authors conclude that is necessary to systematically assess wild species to increase the narrow gene pool of currently grown crops and to significantly intensify molecular studies, including omics approaches, to elucidate the molecular mechanisms underlying heat stress responses and tolerance. They suggest to use optimized transgenic plants to overcome yield losses associated with heat stress.

The effect of short-term moderate heat stress at a highly sensitive stage during reproduction in wheat is reported by Begcy et al. [15]. Authors imposed heat stress for two days at the pollen mitosis stage in four Australian and four European cultivars, and investigated the effects at the physiological and molecular level. In contrast to the Australian cultivars, photosynthetic and transpiration rates as well as pollen viability were strongly reduced in the European cultivars. Moreover, the HSF gene expression pattern showed little variation in the Australian cultivars, while HSFs were either down- or upregulated in the European cultivars. As a conclusion, it appears that European cultivars overreact 
during heat stress, while Australian cultivars are better adapted, showing smaller amplitudes of heat stress responses.

The performance and stability of commercial wheat cultivars under terminal heat stress was studied by Elbasyoni at two locations in Egypt, with the goal to identify the ideal cultivar for each condition [16]. It was shown that heat stress had a significant adverse impact on plant growth and contributed to overall yield losses of about $40 \%$. Based on the findings, it was suggested that importing and evaluating heat-stress-tolerant wheat genotypes under late-sown conditions or heat-stressed conditions is required to boost heat-stress tolerance in adapted wheat cultivars.

Drought stress tolerance can be achieved by generating plants with improved water use efficiency (WUE). Plants with $\mathrm{C}_{3}$ photosynthesis, such as Arabidopsis, are able to moderately increase WUE by restricting transpiration, but this also results in reduced $\mathrm{CO}_{2}$ uptake and thus negatively influences photosynthesis, growth, and yield. In their opinion paper [17], Blankenagel and coworkers discuss recent data obtained for Arabidopsis and the potential to translate the findings to cereals, which are especially drought-sensitive. Their data on the $\mathrm{C}_{4}$ crop maize subjected to progressive drought shows that there is potential for improvement in WUE at the whole plant level, but reduction in assimilation rate and growth trade-offs is even more pronounced compared with Arabidopsis. Authors assume that the $\mathrm{CO}_{2}$ gradient established in $\mathrm{C}_{4}$ metabolism limits the potential for further increases. In conclusion, it is demanded that many more studies are necessary in cereal crops to understand WUE mechanisms under water-deficit conditions.

During growth, plants are not only exposed to multiple abiotic and biotic stresses; access to water and minerals may also be limited simultaneously. Xiong et al. therefore investigated the influence of drought stress and nitrogen supply on the growth of pak choi. Drought was simulated by adding polyethylene glycol under hydroponic culture conditions, resulting in a significant decline in plant water content, transpiration rate, shoot biomass, and shoot nitrogen concentration [18]. Their results further indicated that pak choi supplied with higher nitrogen concentration showed a significant better growth performance under drought stress compared to the controls.

As pointed out already above, in addition to increasing heat and drought stress periods, the duration and frequency of precipitation has significantly increased, often resulting in soil flooding, especially early in the crop-growing season. The review by Mustroph [19] summarizes the progress and approaches to enhancing crop resistance to flooding. With a focus on maize, barley, and soybean, traits such as anatomical and metabolomic adaptations, including aerenchyma formation, adventitious roots, and the formation of a barrier against oxygen loss, but also optimized enzymes and transcriptional regulators, are discussed. Various quantitative trait loci (QTLs) have indicated the presence of tolerance genes. It will now be exciting to identify the corresponding genes and molecular mechanisms, and use the knowledge generated to engineer flooding-tolerant crops.

How natural stress-tolerant plants can be used to understand and exploit tolerance mechanisms is outlined in the opinion paper by Messerer and coworkers [20]. With a focus on salinity-tolerant quinoa, a crop of less economic importance, they highlight recent next-generation sequencing approaches using RNA-seq as a gold standard to identify novel and stress-associated genes. For example, in one study, 15 genes for putative transmembrane proteins were found that could potentially contribute to a higher salinity tolerance. Authors also point out that transcriptomic approaches should be combined with metabolomics to understand quinoa's salinity tolerance. Finally, they suggest applying similar approaches to understand tolerance in other naturally stress-tolerant plants.

\section{Conclusions}

In conclusion, plant sciences currently achieve good models of how model plants react to environmental factors by transcriptional and metabolic reprogramming. However, especially molecular research efforts in crops have to be strengthened considerably. Future biocomputational integration of multiple omics and meta-omics will help further understanding of the complexity of plant stress physiology. This needs further effort in developing innovative research tools and fundamental 
resources for crop plant research, such as reference genomes, proteomes, and metabolomes with comprehensive annotations and structure-function relationships, respectively. Even for the model Arabidopsis, these resources are not fully available. Nevertheless, in several cases, Arabidopsis and other model plants have already been proved suitable for the translation of fundamental research into agronomically relevant crop traits. This is encouraging, but requires further and significant investment into translational research, as pointed out by many authors involved in this Special Issue. Besides this, it remains indispensable to investigate stress resistance mechanisms directly in elite crop plants and in the genetic resources available for breeding.

Author Contributions: T.D. and R.H. both wrote the paper.

Funding: Experimental work in the laboratories of Thomas Dresselhaus and Ralph Hückelhoven is funded by the German Research Foundation DFG in the frame of the Collaborative Research Centre SFB924 and by the Bavarian State Ministry of the Environment and Consumer Protection in the frame of the project network BayKlimaFit.

Conflicts of Interest: The authors declare no conflict of interest.

\section{References}

1. Zhao, C.; Liu, B.; Piao, S.; Wang, X.; Lobell, D.B.; Huang, Y.; Huang, M.; Yao, Y.; Bassu, S.; Ciais, P.; et al. Temperature increase reduces global yields of major crops in four independent estimates. Proc. Natl. Acad. Sci. USA 2017, 114, 9326-9331. [CrossRef] [PubMed]

2. Challinor, A.J.; Watson, J.; Lobell, D.; Howden, S.; Smith, D.; Chhetri, N. A meta-analysis of crop yield under climate change and adaptation. Nat. Clim. Chang. 2014, 4, 287-291. [CrossRef]

3. Schlenker, W.; Roberts, M.J. Nonlinear temperature effects indicate severe damages to US crop yields under climate change. Proc. Natl. Acad. Sci. USA 2009, 106, 15594-15598. [CrossRef] [PubMed]

4. Bender, S.; Butts, M.; Hagemann, S.; Smith, M.; Vereecken, H.; Wendland, F. Einfluss des Klimawandels auf die Terrestrischen Wassersysteme in Deutschland. Eine Analyse Ausgesuchter Studien der Jahre 2009 bis 2013; Report 29; Climate Service Center: Hamburg, Germany, 2017.

5. Schön, C.C. BayKlimaFit-Strategies for the Adaptation of Crop Plants to Climate Change. Available online: http:/ / www.bayklimafit.de (accessed on 25 October 2018).

6. Schwechheimer, C. SFB 924: Molecular Mechanisms Regulating Yield and Yield Stability in Plants. Available online: http:/ / sfb924.wzw.tum.de (accessed on 25 October 2018).

7. Zilber-Rosenberg, I.; Rosenberg, E. Role of microorganisms in the evolution of animals and plants: The hologenome theory of evolution. FEMS Microbiol. Rev. 2008, 32, 723-735. [CrossRef] [PubMed]

8. Aslam, F.; Ali, B. Halotolerant Bacterial Diversity Associated with Suaeda fruticosa (L.) Forssk. Improved Growth of Maize under Salinity Stress. Agronomy 2018, 8, 131. [CrossRef]

9. Lenk, M.; Wenig, M.; Mengel, F.; Häußler, F.; Vlot, A. Arabidopsis thaliana Immunity-Related Compounds Modulate Disease Susceptibility in Barley. Agronomy 2018, 8, 142. [CrossRef]

10. Dawid, C.; Hille, K. Functional Metabolomics-A Useful Tool to Characterize Stress-Induced Metabolome Alterations Opening New Avenues towards Tailoring Food Crop Quality. Agronomy 2018, 8, 138. [CrossRef]

11. Niculaes, C.; Abramov, A.; Hannemann, L.; Frey, M. Plant Protection by Benzoxazinoids-Recent Insights into Biosynthesis and Function. Agronomy 2018, 8, 143. [CrossRef]

12. Ranf, S. Pattern Recognition Receptors-Versatile Genetic Tools for Engineering Broad-Spectrum Disease Resistance in Crops. Agronomy 2018, 8, 134. [CrossRef]

13. Engelhardt, S.; Stam, R.; Hückelhoven, R. Good Riddance? Breaking Disease Susceptibility in the Era of New Breeding Technologies. Agronomy 2018, 8, 114. [CrossRef]

14. Nadeem, M.; Li, J.J.; Wang, M.H.; Shah, L.; Lu, S.Q.; Wang, X.B.; Ma, C.X. Unraveling Field Crops Sensitivity to Heat Stress: Mechanisms, Approaches, and Future Prospects. Agronomy 2018, 8, 128. [CrossRef]

15. Begcy, K.; Weigert, A.; Egesa, A.O.; Dresselhaus, T. Compared to Australian Cultivars, European Summer Wheat (Triticum aestivum) Overreacts When Moderate Heat Stress Is Applied at the Pollen Development Stage. Agronomy 2018, 8, 99. [CrossRef]

16. Elbasyoni, I.S. Performance and Stability of Commercial Wheat Cultivars under Terminal Heat Stress. Agronomy 2018, 8, 37. [CrossRef] 
17. Blankenagel, S.; Yang, Z.; Avramova, V.; Schön, C.C.; Grill, E. Generating plants with improved water use efficiency. Agronomy 2018, 8, 194. [CrossRef]

18. Xiong, X.; Chang, L.Y.; Khalid, M.; Zhang, J.J.; Huang, D.F. Alleviation of Drought Stress by Nitrogen Application in Brassica campestris ssp. Chinensis L. Agronomy 2018, 8, 66. [CrossRef]

19. Mustroph, A. Improving flooding tolerance of crop plants. Agronomy 2018, 8, 160. [CrossRef]

20. Messerer, M.; Lang, D.; Mayer, K.F.X. Analysis of Stress Resistance Using Next Generation Techniques. Agronomy 2018, 8, 130. [CrossRef]

(C) 2018 by the authors. Licensee MDPI, Basel, Switzerland. This article is an open access article distributed under the terms and conditions of the Creative Commons Attribution (CC BY) license (http:/ / creativecommons.org/licenses/by/4.0/). 